\title{
Effects and mechanism of downregulation of COX-2 expression by RNA interference on proliferation and apoptosis of human breast cancer MCF-7 cells
}

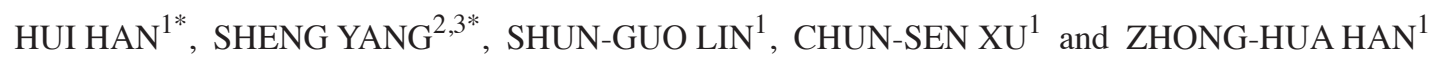 \\ Departments of ${ }^{1}$ Breast Surgery and ${ }^{2}$ Medical Oncology, The Union Hospital of Fujian Medical University; \\ ${ }^{3}$ Fujian Provincial Key Laboratory of Tumor Translational Medicine, Fuzhou, Fujian 350001, P.R. China
}

Received November 4, 2013; Accepted July 9, 2014

DOI: $10.3892 / \mathrm{mmr} .2014 .2659$

\begin{abstract}
The aim of the present study was to investigate the effects of RNA interference with prostaglandin-endoperoxide synthase 2 (COX-2) gene on the proliferation and apoptosis of breast cancer MCF-7 cells, as well as the underlying mechanism. The present study constructed the eukaryotic expression vector of the targeted COX-2 gene, transfected the MCF-7 cells and screened the stably expressed clone. Changes in the COX-2 gene expression in breast cancer MCF-7 cells prior to and following transfection were examined; the proliferation and apoptosis of MCF-7 cells were analyzed. Furthermore, changes in the protein levels of survivin, B-cell lymphoma 2 (Bcl-2) and Bcl-2-associated X (Bax) genes were detected. RNA interference mediated by a lentiviral expression vector significantly decreased the protein expression levels of the COX-2 gene, and therefore, the proliferation and growth of breast cancer MCF-7 cells was significantly suppressed and the apoptotic rate increased. Of note, the mRNA and protein expression levels of survivin and Bcl-2 decreased, while those of Bax increased following COX-2 silencing. RNA interference markedly deactivated the COX-2 gene, suppressed the proliferation of breast cancer MCF-7 cells, and, to a certain extent, enhanced the induced spontaneous apoptosis, which is regulated by the Bax gene. These results provided evidence for the potential applications of RNA interference of the targeted COX-2 gene in gene therapy for the treatment of breast cancer.
\end{abstract}

Correspondence to: Dr Hui Han, Department of Breast Surgery, The Union Hospital of Fujian Medical University, 29 Xinquan Road, Fuzhou, Fujian 350001, P.R. China

E-mail: 939807@sohu.com

${ }^{*}$ Contributed equally

Key words: RNA interference, breast cancer, proliferation, apoptosis, prostaglandin-endoperoxide synthase 2

\section{Introduction}

At present, breast cancer is one of the most common malignant types of cancer in females worldwide and continues to be one of the main diseases threatening the health of females $(1,2)$. The underlying mechanism, prevention and treatment of breast cancer, however, remains poorly understood, and basic and effective measures for its treatment are lacking worldwide. Although marked achievements have been made in comprehensive treatment, the general curative effect remains to be improved. With the progression in the fields of the molecular biology of cancer and associated disciplines, gene therapy of breast cancer is considered to be a novel treatment option alongside the traditional methods, including surgery, radiotherapy, chemotherapy and endocrinotherapy $(3,4)$. RNA interference (RNAi), an evolutionarily-conserved cellular defense mechanism mediated by small interfering RNA, stably, specifically and effectively initiates gene silencing with low cytotoxicity following transcription, and is therefore a method that has been widely applied in studies on functional genome and gene therapy $(5,6)$. Prostaglandin-endoperoxide synthase 2 (COX-2), an inductive enzyme that is not expressed, or expressed at markedly low levels, in normal tissues, and which is increased in pathological states, has been closely associated with the development of breast cancer $(7,8)$. The present study successfully constructed the lentiviral expression vector of the short hairpin RNA (shRNA) of the targeted COX-2 gene, which was then applied silence COX-2 in the breast cancer cell line MCF-7, allowing the investigation of the mechanism and effect of COX-2 on the proliferation and apoptosis of the MCF-7 cells.

\section{Materials and methods}

Materials. The breast cancer cell lines MCF-7 and 293T were purchased from Shanghai Cell Bank (Chinese Academy of Science, Shanghai, China). pSPAX2, pMD2G and pLVX-shRNA1 vectors were from Clontech (Mountain View, CA, USA), and plasmid DNA extraction kits (Qiagen, Valencia, CA, USA), Opi-MEM, Escherichia coli DH5 $\alpha$ and Taq DNA polymerase (Invitrogen Life Technologies, Carlsbad, CA, USA), T4 DNA ligase, BamHI and EcoRI 
restriction endonuclease (New England Biolabs, Ipswich, MA, USA), liposome Lipofectamine 2000, Dulbecco's modified Eagle's medium (DMEM) culture medium, fetal calf serum and trypsin (Invitrogen Life Technologies), gel retrieve kits (Qiagen, Hilden, Germany), KOD hi-fi enzyme PCR kits (Toboyo, Osaka, Japan), Taq enzyme (Toyobo, Osaka, Japan) and DNA ladder (Fermentas, Burlington, ON, Canada) were used.

Cell culture. The breast cancer MCF-7 cells were cultured in DMEM containing $10 \%$ fetal calf serum, which was placed in a thermostat with $5 \% \mathrm{CO}_{2}$ at $37^{\circ} \mathrm{C}$. The cells were digested and passaged with $0.25 \%$ lipase every $2-3$ days, and cells in the exponential growth phase were selected to be used in the experiments.

Design and screening of shRNA of targeted COX-2. Based on the COX-2 mRNA sequence in Genbank and the principles of shRNA design, the target sequence and three pairs of COX-2-targeting shRNA were designed (Table I). The shRNAs were synthesized by Shanghai Hanbio Co., Ltd. (Shanghai, China), and then transfected into 293T cells with Lipofectamine 2000 according to the manufacturer's instructions; the results were observed under a fluorescence microscope (BX50; Olympus, Tokyo, Japan) $24 \mathrm{~h}$ following transfection, and the cell collection and protein abstraction were conducted $36 \mathrm{~h}$ thereafter. The most efficient shRNA was selected via western blot analysis.

Construction and transfection of lentiviral expression vector of COX-2-shRNA. The most efficient shRNA was selected as the interference target out of the three pairs in Table I. A double-stranded DNA fragment was synthesized in vitro, with cohesive termini of BamHI and EcoRI restriction enzymes and an internal hairpin sequence of 5'-CCATTCTCCTTGAAAGGACTTTTCAAG AGAAAGTCCTTTCAAGGAGAATGG-3'. The fragment was ligated into pGC-LV and transfected with Escherichia coli DH5 $\alpha$. Following amplification, screening and selection, the positive clone was sequenced by Invitrogen Life Technologies. The plasmid was extracted and the lentiviral expression vector of COX-2-shRNA was recombined. The breast cancer MCF-7 cells transfected with the COX-2-shRNA lentiviral vector were defined as group COX-2-shRNA; those transfected with the empty vector and those without any treatment were named as the mock and blank groups, respectively.

Extraction of general RNA and quantitative polymerase chain reaction ( $q P C R$ ) detection. The general RNA of each cell group was extracted with TRIzol, reverse-transcribed into cDNA and subjected to qPCR according to the manufacturers' instructions of the KOD hi_fi enzyme PCR kit (Toboyo) and the PCR kit supplied by Invitrogen Life Technologies. The COX-2 and GAPDH primers (as the internal control) were synthesized by Shanghai Hanbio Co., Ltd. As for COX-2, the forward primer was 5-CCCTTGG GTGTCAAAGGTAA-3', while the reverse primer was 5'-GCCCTCGCTTATGATCTGTC-3'; for GAPDH, the forward primer was 5'-AGAAAATCTGGCACCACACC-3' while the reverse was 5'-AGAGGCGTACAGGGATAGCA-3'.
Table I. COX-2-shRNA sequence.

\begin{tabular}{lc}
\hline shRNA number & Sequence \\
\hline COX-2 shRNA-1 & GCTGAATTTAACACCCTCTAT \\
COX-2 shRNA-2 & GCAGATGAAATACCAGTCTTT \\
COX-2 shRNA-3 & CCATTCTCCTTGAAAGGACTT
\end{tabular}

COX-2, prostaglandin-endoperoxide synthase 2; shRNA, small hairpin RNA.

The reaction conditions of the two-step PCR were: 45 cycles of pre-denaturation at $95^{\circ} \mathrm{C}$ for $15 \mathrm{sec}$, denaturation at $95^{\circ} \mathrm{C}$ for $15 \mathrm{sec}$ and annealing at $60^{\circ} \mathrm{C}$ for $30 \mathrm{sec}$. Following PCR, the mixture was denatured for $1 \mathrm{~min}$ at $95^{\circ} \mathrm{C}$, cooled to $55^{\circ} \mathrm{C}$ at which the double strands of DNA combined sufficiently. From $55-95^{\circ} \mathrm{C}$, each elevation of $0.5^{\circ} \mathrm{C}$ was kept for $4 \mathrm{sec}$. The light absorption value was recorded using an absorption reader (AG 22331; Eppendorf, Hamburg, Germany) and the melting curve was plotted. Through the $2^{-\Delta \Delta \mathrm{Ct}}$ method, quantitative analysis was performed with the ratio of the target gene to GAPDH.

Detection of protein expression by western blot analysis. A total of $72 \mathrm{~h}$ following transfection, the cells were collected; the general proteins were extracted, quantified by the bicinchoninic acid assay and normalized. A standard curve of absorbance, vs. micrograms protein was constructed and vice versa. This was used to determine the concentrations of the original samples from the quantity of protein, volume/sample, and dilution factor

(Thermo Fisher Scientific, Rockford, IL, USA). The sample load was $30 \mu \mathrm{g}$ extracted protein per lane. Protein purified by $10 \%$ SDS-PAGE was transferred to a polyvinylidene fluoride membrane following electrophoresis. The protein was blocked with $5 \%$ skimmed milk powder at $4{ }^{\circ} \mathrm{C}$. The primary monoclonal antibodies (Cell Signaling Technology, Inc., Danvers, MA, USA), COX-2 (1:500), survivin (1:800), B-cell lymphoma 2 (Bcl-2) (1:800), Bcl-2-associated X (Bax) (1:800) and GAPDH (1:4,000), were added and the mixture was subsequently incubated overnight at $4^{\circ} \mathrm{C}$ with agitation. Following washing the membrane for $10 \mathrm{mins}$ three times, the horseradish peroxidase-conjugated antibody II $(1: 4,000)$ was added for a 2 -h incubation. The protein bands were detected (the colored membranes) using an enhanced chemiluminescence (ECL) system (Pierce Biotechnology, Inc., Rockford, IL, USA) and exposed to an X-ray film. The gray membranes were scanned with the image analytical system (Image J software, National Institutes of Health, Bethesda, MD, USA).

Detection of cell proliferation by MTT assay. The breast cancer MCF-7 cells at exponential growth phase were inoculated into the 96 -well plates $\left(100 \mu \mathrm{l} /\right.$ well, $1 \times 10^{4} /$ well $)$ inside a thermostat at $37^{\circ} \mathrm{C}$ with $5 \% \mathrm{CO}_{2}$ and saturated humidity. An MTT assay, in which $20 \mu \mathrm{l}$ MTT reagent was added and then placed in a $\mathrm{CO}_{2}$ incubator for $4 \mathrm{~h}$ (Sigma, St. Louis, MO, 
A

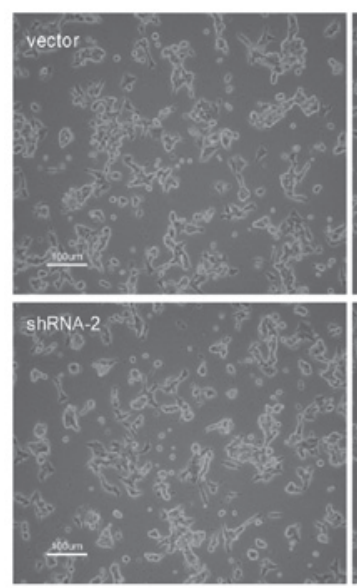

B

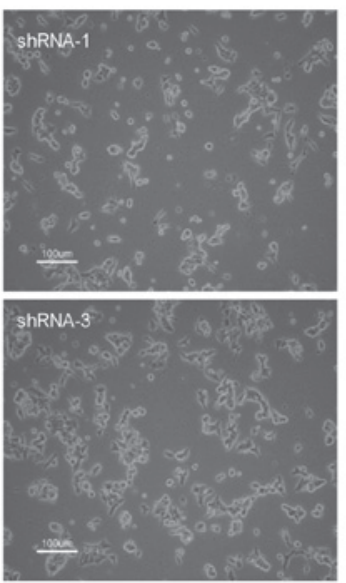

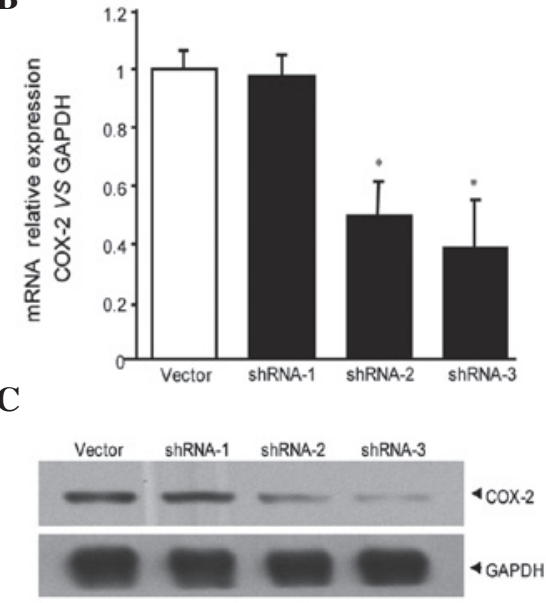

Figure 1. (A) Empty vector as well as COX-2-shRNA-1, 2, 3 plasmids were effectively transfected into the breast cancer MCF-7 cells (magnification x100). (B) The efficiency of COX-2 interference was assessed by quantitative polymerase chain reaction ( $\mathrm{P}<0.05$, vs. vector). (C) Efficiency of COX-2 interference detected by western blot analysis. COX-2, prostaglandin-endoperoxide synthase 2; shRNA, small hairpin RNA.

USA) was performed following incubation for 2, 24, 48 and $72 \mathrm{~h}$, respectively. The optical density (OD) value at $570 \mathrm{~nm}$ wavelength was detected with a microplate spectrophotometer (Type 550; R\&D Systems, Minneapolis, MN, USA). The mean value of five wells was the final OD value. The cell proliferation curve was plotted with the time on the horizontal axis and the OD value on the vertical axis. The growth inhibition rate of cancer cells was calculated using the following formula: Growth inhibition rate $=(1-$ OD value of COX-2-shRNA/OD value of blank) x 100 .

Detection of cancer cell apoptosis via flow cytometry. The breast cancer MCF-7 cells of each group were digested and centrifuged for $5 \mathrm{~min}$ with the supernatants discarded. The cells were then washed with cold phosphate-buffered saline (PBS) and suspended again in $100 \mu \mathrm{l}$ binding buffer solution. The cell concentration was adjusted to $1 \times 10^{6} / \mathrm{ml}, 2 \mu \mathrm{l}$ of the Annexin V-fluorescein isothiocyanate (FITC; $20 \mu \mathrm{g} / \mathrm{ml}$ ) was added and the sample was thoroughly mixed. The mixture was incubated in the dark at room temperature for $15 \mathrm{~min}$ and then diluted with $400 \mu \mathrm{l}$ PBS. 2 min prior to flow cytometric analysis (FACSCalibur; Becton-Dickinson, Franklin Lakes, NJ, USA), $1 \mu \mathrm{l}$ propidium iodide (PI) $(50 \mu \mathrm{g} / \mathrm{ml})$ was added. Another sample without Annexin V-FITC or PI served as a negative reference.

Statistical analysis. The data were processed by SPSS 16.0 statistical software (SPSS, Inc., Chicago, IL, USA). Quantitative data were expressed as the mean \pm standard deviation. The significant difference among the groups was assessed using analysis of variance. $\mathrm{P}<0.05$ was considered to indicate a statistically significant difference.

\section{Results}

Screening outcome of COX-2-shRNA lentiviral expression vectors. As shown in Fig. 1A, the vectors for COX-2-targeting shRNAs, COX-2-shRNA-1,2 and 3, were effectively transfected into the MCF-7 cells, as demonstrated by green fluorescence.
A

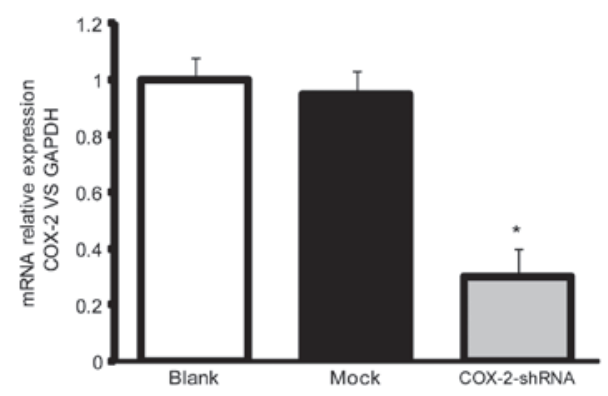

B

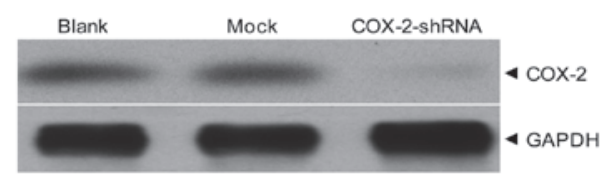

Figure 2. (A) The COX-2 mRNA expression in the breast cancer cell line MCF-7 was significantly decreased in the COX-2-shRNA-transfected group. (B) The COX-2 protein expression in MCF-7 cells decreased eminently in the COX-2-shRNA-transfected group. COX-2, prostaglandin-endoperoxide synthase 2; shRNA, small hairpin RNA.

The outcomes of the qPCR and western blot analyses demonstrated that shRNA-1 had no evident interference effect; shRNA-2 exerted a significant effect, and shRNA-3 was most efficient at downregulating COX-2 (Fig. 1B and C). Therefore, the expression vector of the shRNA plasmid was recombined based on the shRNA-3 sequence. This COX-2-shRNA plasmid was transfected into the MCF-7 cells and screened and amplified by G418 for further study.

Effects of COX-2-shRNA on COX-2 mRNA in MCF-7 cells. qPCR analysis suggested that following transfection of MCF-7 cells with the RNAi expression vector, the COX-2 mRNA expression of the breast cancer MCF-7 cells in COX-2-shRNA was significantly lower than that in the blank and mock groups $(\mathrm{P}<0.05)$, while there was no significant difference among the blank and mock groups (Fig. 2A). Results of the western blot analysis were consistent with those 


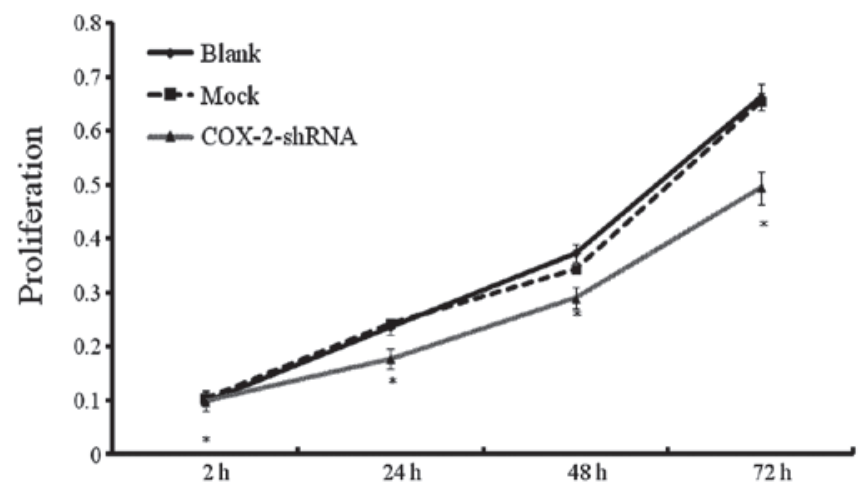

Figure 3. The MTT assay demonstrates that the cell growth rate in COX-2-shRNA-transfected cells declines evidently compared with that in the blank and mock-transfected groups. " $\mathrm{P}<0.05$ compared with the mock and blank groups. COX-2, prostaglandin-endoperoxide synthase 2; shRNA, small hairpin RNA.

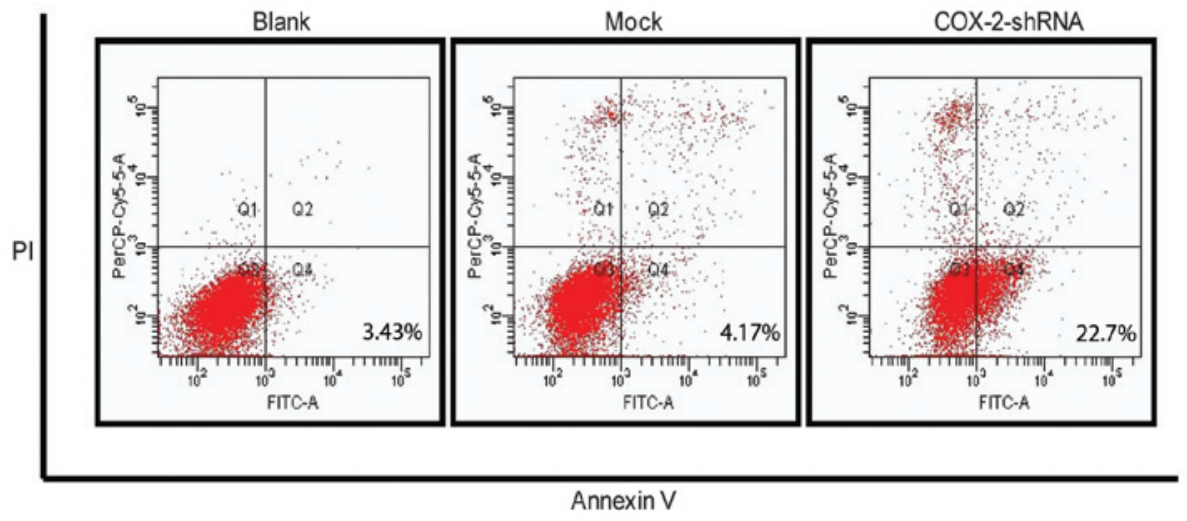

Figure 4. Annexin X and PI staining followed by flow cytometric analysis indicates that the rate of apoptosis of breast cancer MCF-7 cells transfected with COX-2-shRNA is evidently higher than that of blank or mock-transfected cells. COX-2, prostaglandin-endoperoxide synthase 2; shRNA, small hairpin RNA; PI, propidium iodide; FITC, fluorescein isothiocyanate..

of the $\mathrm{qPCR}$, as the protein expression decreased eminently in the COX-2-shRNA group as compared with that in the blank and mock groups (Fig. 2B).

Effects of COX-2-shRNA on the proliferation and growth of breast cancer MCF-7 cells. The cell growth curves were plotted based on the OD values of the COX-2-shRNA, blank and mock groups. The original OD values for these three groups were $0.0986 \pm 0.0076,0.0994 \pm 0.0186$ and $0.1037 \pm 0.0134$, respectively, and were not significantly different from each other $(\mathrm{P}>0.05)$. On Day three, however, the OD values were $0.4949 \pm 0.0308,0.6628 \pm 0.0245$ and $0.6545 \pm 0.0155$, with no significant difference between the blank and mock groups $(\mathrm{P}>0.05)$. The cell growth rate in the COX-2-shRNA group had declined evidently compared with that in the blank and mock groups $(\mathrm{P}<0.05)$, with growth inhibition rates of 24.47 , 22.19 and $25.34 \%$ at 24,48 and $72 \mathrm{~h}$ following COX-2 gene interference (Fig. 3).

Effects of COX-2-shRNA on apoptosis of MCF-7 cells. Flow cytometric analysis indicated that the apoptotic rates were $3.43 \pm 0.90$ and $4.17 \pm 1.33 \%$ in the mock and blank groups, respectively, demonstrating no significant difference $(\mathrm{P}>0.05)$, while that in the COX-2-shRNA group was $22.70 \pm 4.33 \%$, which was significantly higher than that of the other groups ( $\mathrm{P}<0.01$; Fig. 4).
Changes in survivin, Bcl-2 and Bax levels following COX-2 gene interference. $\mathrm{qPCR}$ analysis demonstrated that following transfection, the mRNA expression levels of survivin and $\mathrm{Bcl}-2$ in the COX-2-shRNA group were significantly lower than those in the blank and mock groups $(\mathrm{P}<0.05)$, while the mRNA expression of Bax was higher $(\mathrm{P}<0.05)$. No significant difference was observed in the expression of survivin, Bcl-2 and Bax between the blank and mock groups $(\mathrm{P}>0.05$; Fig. 5A). In terms of protein expression of survivin, Bcl-2 and Bax, the western blot analysis revealed results consistent with those of the qPCR (Fig. 5B).

\section{Discussion}

At present, the incidence rate of breast cancer in western developed countries remains at a stable high level, ranking first among the various types of female malignant tumor; in Asia, particularly in Japan, Singapore and the coastal cities of China, the rate is increasing $(9,10)$. The pathogenesis of breast cancer, however, has not been completely elucidated. Although novel anti-cancer drugs are constantly emerging and the existing strategies are being improved, the efficacy and safety are not sufficient. The prevention and treatment lack basic and effective measures, and therefore, further studies on breast cancer prevention and treatment are required $(11,12)$. The modern molecular biology of cancer demonstrates that the onset and development 

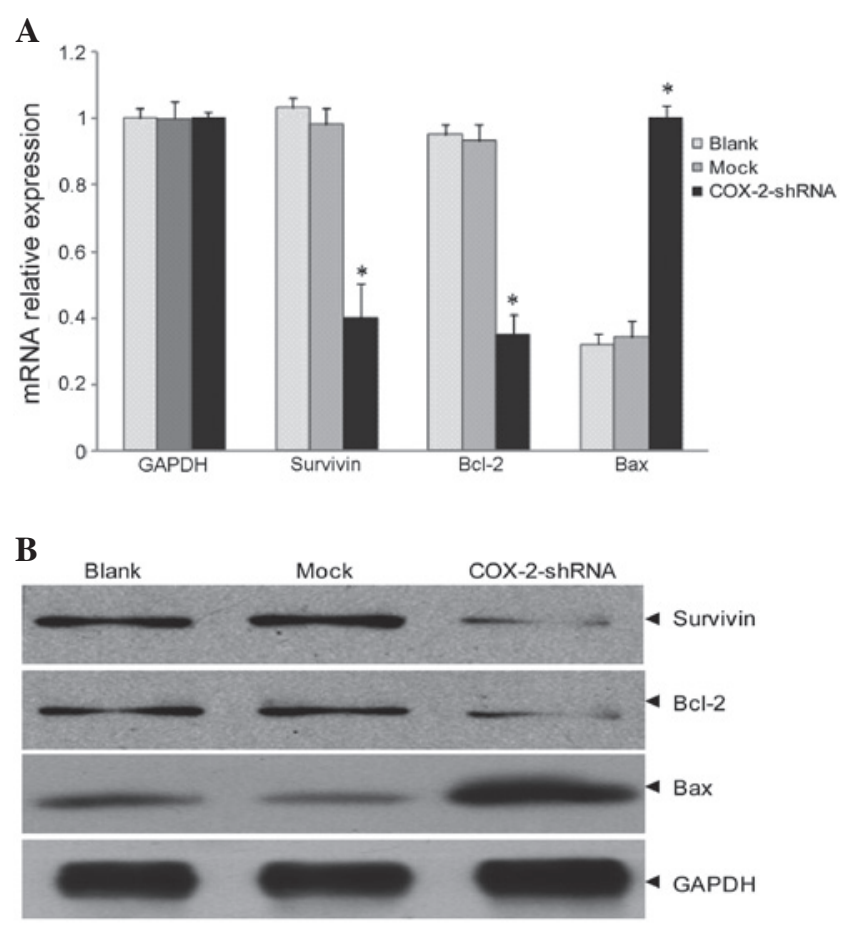

Figure 5. (A) The mRNA expression of survivin and Bcl-2 in COX-2-shRNAtransfected cells was significantly lower, while the mRNA expression of Bax was higher than that in the blank or mock groups ( ${ }^{*} \mathrm{P}<0.05$, vs. blank group). (B) The protein expression of survivin and Bcl-2 in COX-2-shRNAtransfected cells was significantly decreased, while the mRNA expression of Bax was higher than that in the blank or mock groups. COX-2, prostaglandin-endoperoxide synthase 2; shRNA, small hairpin RNA; Bcl-2, B-cell lymphoma 2; Bax, Bcl-2-associated X.

of breast cancer constitute a complex biological process with participation of multiple genes, function of multiple factors and composition of multiple stages. Breast cancer is a genetic disease, and therefore, the activation of a cancer gene or the deactivation of an anti-cancer gene leads to the abnormal proliferation, differentiation and apoptosis of cells. It is therefore the key mechanism underlying the onset and development of breast cancer, and one of the important mechanisms of the drug resistance of cancer cells. Therefore, studies investigating novel cell targets to treat cancer, and in particular, further improving the clinical curative effect of breast cancer treatment, are gaining increasing attention $(13,14)$.

Numerous studies have demonstrated that COX-2 is closely associated with the onset of cancer and its probable function in breast cancer development has been attracting significant attention $(15,16)$. The correlation between COX-2 and breast cancer was first revealed in 1983 by Bennett et al (17), who discovered that the prostaglandin (PG), the product of the enzymatic reaction of COX-2, was higher in breast cancer tissues than in normal tissues. Spizzo et al (18) identified that the positive expression rate of COX-2 in 212 breast cancer samples was 48.6\%. Denkert et al (19) further revealed the associations between COX-2 expression and high nuclear grade, enlarged tumors, negative state of estrogen receptor (ER) and progesterone receptor (PR), and HER-2 gene amplification. The study also revealed that COX-2 is closely correlated with lymphatic metastasis. Previous studies $(20,21)$ with large samples supported the evidence that COX-2 is highly expressed in breast cancer, differently in benign tumors, and not at all or at low levels in normal tissues, which fully demonstrated the close correlation between COX-2 and breast cancer. Numerous studies have revealed the complex mechanism of COX-2 inducing breast cancer: The overexpression of COX-2 increases PG product, which stimulates cell proliferation leading to the formation of a tumor $(22,23)$; COX-2 overexpression also leads to apoptosis resistance in cancer cells, while the suppression of its activity induces apoptosis (24). Through catalysis, COX-2 overexpression produces more prostaglandin E2 (PGE2) and inhibit the anti-tumor immunity to facilitate the growth of cancer cells (25). Singh and Lucci (26) proved that with COX-2 overexpression in breast cancer, PGE2 inhibits the functions of dendritic cells (DCs), and thus significantly reduces the expression of the co-stimulation molecules B7 and CD40, therefore preventing DC phagocytosis and antigen presence, improving the tumor's immunotolerance and facilitating its escape from immunological surveillance. COX-2 regulates the cell cycle, as its continuous over-expression is followed by an evident prolongation of the G1 phase and decreased activity of cyclin D1, retinoblastoma protein 1 kinase and cyclin-dependent kinase 4 , thus prohibiting apoptosis. The prolongation of the cell cycle strengthens the adherence to the extracellular matrix, while abnormal prolongation causes a series of mutations and telomutations, which promote the formation of tumors. Furthermore, COX-2 functions in strengthening the attack and metastasis of the tumor, assisting the formation of tumor vessels, and enhancing the drug resistance characteristics of cancer cells (27-29).

In the present study, qPCR and western blot analyses were adopted to prove that following transfection of breast cancer MCF-7 cells with a COX-2-shRNA lentiviral vector, the COX-2 transcription and translation were significantly decreased, indicating the successful construction of the COX-2-shRNA lentiviral vector. The results suggested that in the COX-2-shRNA group, the expression of COX-2 mRNA and protein were lowered significantly and the proliferation and growth of breast cancer MCF-7 cells were notably suppressed. Flow cytometric analysis demonstrated that the apoptotic rate of MCF-7 cells was evidently increased. A study by Kundu et al (30) indicated that the growth inhibition of breast cancer cell strains induced by a COX-2 suppressor was associated with a change in the cell morphology. This change was recovered following medicine withdrawal. Compared with the blank and mock groups, the cell count in the G0/G1 phase significantly increased and that in the S phase decreased. This result indicated the importance of the COX-2 gene in the transition of MCF-7 cells from G1 phase to S phase, and that following silencing COX-2 expression, the suppression of cell proliferation should be the result of both an increase in apoptosis and a loss in function of promoting cell division. COX-2 overexpression may lead to apoptosis resistance of cancer cells and the suppression of COX-2 activity may induce apoptosis, which has been proved in numerous different transfection studies of cancer cells. In numerous cases, apoptosis suppression is another mechanism promoting the proliferation and growth of cancer cells, but the anti-apoptosis of COX-2 has remained to be fully elucidated $(31,32)$. The majority of studies agree on the following aspects: (i) COX-2 increases the expression of Bcl-2, which is a key anti-apoptosis and anti-oxidation protein. The expression of the apoptosis proteins Bax and 
Bcl-2-extra large are decreased through the suppression of lipid over-oxidation. Studies have confirmed that the Bcl-2 expression may be lowered by a COX-2 suppressor. In the cancer tissues of transgenic rats, O'Mahony et al (33) observed that the over-expression of COX-2 caused both a significant reduction of apoptosis and changes in levels of anti-apoptotic and pro-apoptotic proteins; (ii) COX-2 reduced apoptosis through weakening and suppressing the nitric oxide signaling pathway; (iii) COX-2 suppressed apoptosis via decreasing sphingosine. Furthermore, Orlov et al (34) discovered that the prostaglandin compounds, which are products of COX-2mediated reactions, increase the concentration of cyclic adenosine monophosphate (cAMP), which leads to the apoptosis-resistance of cancer cells, while a COX-2 suppressor decreased cell division and increased apoptosis. In the present study, the expression of COX-2 mRNA and protein in the COX-2-shRNA group was decreased significantly, and levels of survivin and Bcl-2 were greatly reduced, while Bax levels were increased. Therefore, one of the main anti-breast cancer mechanisms of the silencing of COX-2 expression by RNA interference is considered to be the suppression of survivin and Bcl-2 and the increase of Bax gene activity, which led to the decrease of apoptosis resistance of MCF-7 cells and the increase in sensitivity of breast cancer cells to apoptotic factors $(35,36)$.

In conclusion, the present study proved that RNA interference effectively silenced the expression of the COX-2 gene, suppressed proliferation and induced apoptosis of breast cancer cells, with the important function of controlling the activity of apoptosis-associated genes. The specific mechanism underlying this effect requires further study, but clinically, this evidence may have extensive applications for the development of novel strategies in COX-2 targeted gene therapy for cancer $(37,38)$.

\section{Acknowledgements}

The present study was supported by grants from the Youth Science \& Research Project of the Health Department of Fujian Province (grant no. 2009-2-23).

\section{References}

1. Parkin DM, Bray F, Ferlay J, et al: Global cancer statistics 2002. CA Cancer J Clin 55: 74-108, 2005.

2. Lazzeroni $M$ and Decensi A: Breast cancer prevention by antihormones and other drugs: where do we stand? Hematol Oncol Clin North Am 27: 657-672, 2013.

3. Singel SM, Cornelius C, Batten K, et al: A targeted RNAi screen of the breast cancer genome identifies KIF14 and TLN1 as genes that modulate docetaxel chemosensitivity in triple-negative breast cancer. Clin Cancer Res 19: 2061-2070, 2013.

4. Zheng WY, Kang YY, Li LF, et al: Levels of effectiveness of gene therapies targeting survivin and its splice variants in human breast cancer cells. Drug Discov Ther 5: 293-238, 2011.

5. Ashihara E: RNA interference for cancer therapies. Gan To Kagaku Ryoho 37: 2033-2041, 2010.

6. Liu JL, Wei W, Tang W, et al: Silencing of lysyl oxidase gene expression by RNA interference suppresses metastasis of breast cancer. Asian Pac J Cancer Prev 13: 3507-3511, 2012.

7. Timoshenko AV, Chakraborty C, Wagner GF, et al: COX-2-mediated stimulation of the lymphangiogenic factor VEGF-C in human breast cancer. Br J Cancer 4: 1154-1163, 2006.

8. Dhakal HP, Naume B, Synnestvedt M, et al: Expression of cyclooxygenase-2 in invasive breast carcinomas and its prognostic impact. Histol Histopathol 27: 1315-1325, 2012.
9. Troisi R, Altantsetseg D, Davaasambuu G, et al: Breast cancer incidence in Mongolia. Cancer Causes Control 23: 1047-1053, 2012.

10. Chuang E, Paul C, Flam A, et al: Breast cancer subtypes in Asian-Americans differ according to Asian ethnic group. J Immigr Minor Health 14: 754-758, 2012.

11. Engebraaten O, Moen Vollan HK and Børresen-Dale AL: Triple-Negative Breast Cancer and the Need for New Therapeutic Targets. Am J Pathol pii: S0002-S9440, 00461-00466, 2013.

12. De Los Santos JF, Cantor A, Amos KD, et al: Magnetic resonance imaging as a predictor of pathologic response in patients treated with neoadjuvant systemic treatment for operable breast cancer. Translational Breast Cancer Research Consortium trial 017. Cancer 119: 1776-1183, 2013.

13. Brim RL and Pearson SD: The use and reporting of patient-reported outcomes in phase III breast cancer trials. Clin Trials 10: 243-249, 2013.

14. Arthur DW, Vicini FA, Todor DA, et al: Contura Multi-Lumen Balloon breast brachytherapy catheter: comparative dosimetric findings of a phase 4 trial. Int J Radiat Oncol Biol Phys 86: 264-269, 2013.

15. Holmes MD, Chen WY, Schnitt SJ, et al: COX-2 expression predicts worse breast cancer prognosis and does not modify the association with aspirin. Breast Cancer Res Treat 130: 657-662, 2011.

16. Singh AK, Parshad R, Pasi S, et al: Prognostic significance of cyclooxygenase- 2 and response to chemotherapy in invasive ductal breast carcinoma patients by real time surface plasmon resonance analysis. DNA Cell Biol 30: 801-807, 2011.

17. Bennett A, Berstock DA, Carroll MA, et al: Breast cncer, its recurrence, and patient survival in relation to tumor prostaglandins. Adv Prostaglandin Thromboxane Leukot Res 12: 299-302, 1983

18. Spizzo G, Gastl G, Wolf D, et al: Correlation of COX-2 and Ep-CAM over expression in human invasive breast cancer and its impact on survival. Br J Cancer 88: 574-578, 2003.

19. Denkert C, Winzer KJ and Hauptmann S: Prognostic impact of cyclooxygenase-2 in breast cancer. Clin Breast Cancer 4: 428-433, 2004.

20. Dhakal HP, Naume B, Synnestvedt M, et al: Expression of cyclooxygenase-2 in invasive breast carcinomas and its prognostic impact. Histol Histopathol 27: 1315-1325, 2012.

21. Ristimäki A, Sivula A, Lundin J, et al: Prognostic significance of elevated cyclooxygenase-2 expression in breast cancer. Cancer Res 62: 632-635, 2002.

22. Oliveira VM, Piato S and Silva MA: Correlation of cyclooxygenase-2 and aromatase immunohistochemical expression in invasive ductal carcinoma, ductal carcinoma in situ, and adjacent normal epithelium. Breast Cancer Res Treat 95: 235-241, 2006.

23. Takafuji VA, Evans A, Lynch KR and Roche JK: PGE(2) receptors and synthesis in human gastric mucosa: perturbation in cancer. Prostaglandins. Leukot Essent Fatty Acids 66: 71-81, 2002

24. Khor LY, Bae K, Pollack A, et al: COX-2 expression predicts prostate-cancer outcome: analysis of data from the RTOG 92-02 tria1.Lancet Oncol 8: 912-920, 2007

25. Pazhang Y, Ahmadian S, Javadifar N, et al: COX-2 and survivin reduction may play a role in berberine-induced apoptosis in human ductal breast epithelial tumor cell line. Tumour Biol 33: 207-214, 2012.

26. Singh B and Lucci A: Role of cyclooxygenase- 2 in breast cancer. J Surg Res 108: 173-179, 2002.

27. Sivula A, Talvensaari-Mattila A, Lundin J, et al: Association of cyclooxygenase- 2 and matrix metalloproteinase-2 expression in human breast cancer. Breast Cancer Res Treat 89: 215-220, 2005.

28. Uefuji K, Ichikura T and Mochizuki H: Cyclooxygenase-2 expression is related to prostagland in biosynthesis and angiogenes is in gastric cancer. Clin Cancer Res 6: 135-138, 2000.

29. Surowiak P, Materna V, Matkowski R, et al: Relationship between the expression of cyclooxygenase-2 and MDR1/P2glycoprotein in invasive breast cancers and their prognostic significance. Breast Cancer Res 7: R862-R870, 2005.

30. Kundu N, Smyth MJ, Samsel L, et al: Cyclooxygenase inhibitors block cell growth, increase ceramide and inhibit cell cycle. Breast Cancer Res Treat 76: 57-64, 2002.

31. Salhab M, Singh-Ranger G, Mokbel R, et al: Cyclooxygenase-2 mRNA expression correlates with aromatase expression in human breast cancer.J Surg Oncol, 96: 424-8, 2007 
32. Basu GD, Pathangey LB, Tinder TL, et al: Cyclooxygenase-2 inhibitor induces apoptosis in breast cancer cells in an in vivo model of spontaneous metastatic breast cancer. Mol Cancer Res 2: 632-642. 2004

33. O'Mahony CA, Beauchamp RD, Albo D, et al. Cyclooxygenase 2 alters transforming growth factor $\beta 1$ response during intestinal tumorigenesis. Surgery 126: 364-370, 1999

34. Orlov SN, Thorin-Trescases N, Dulin NO, et al: Activation of cAMP signaling transiently inhibits apoptosis in vascular smooth muscle cells in a site upstream of caspase-3. Cell Death Differ 6: 661-672,1999

35. Cao Y, Pearman AT, Zimmerman GA, et al: Intracellular unesterified arachidonic acid signals apoptosis. Proc Natl Acad Sci USA 7: 11280-1128, 2000.
36. Cao Y and Prescott SM: Many actions of cyclooxygenase-2 in cellular dynamics and in cancer. J Cell Physiol 190: 279-286, 2002.

37. Yousif S, Chaves C, Potin S, et al: Induction of P-glycoprotein and Bcrp at the rat blood-brain barrier following a subchronic morphine treatment is mediated through NMDA/COX-2 activation. J Neurochem 123: 491-503, 2012.

38. Jana D, Sarkar DK, Maji A, et al: Can cyclo-oxygenase-2 be a useful prognostic and risk stratification marker in breast cancer? J Indian Med Assoc 110: 429-433, 2012. 